\title{
Observation of Thermally-Activated Electron Traps in GaAs/AlAs/GaAs Heterostructures in Low-Frequency Noise Measurements
}

\author{
J. Przybytek ${ }^{a}$, R. Stankiewicz ${ }^{a}$, M. Gryglas-Borysiewicz ${ }^{a}$, M. Baj $^{a}$, A. Cavanna $^{b}$ \\ AND G. FAINI ${ }^{b}$ \\ ${ }^{a}$ Institute of Experimental Physics, Faculty of Physics, University of Warsaw, Hoża 69, 00-681 Warsaw, Poland \\ ${ }^{b}$ Laboratoire de Photonique et Nanostructures, CNRS, Marcoussis, France
}

\begin{abstract}
During our investigations of tunneling process in thin $7 \mathrm{~nm}$ thick GaAs/AlAs/GaAs vertical single-barrier tunneling structure with Si $\delta$-doping inside the barrier we have observed fluctuations of the tunneling current which exhibited large Lorentzian noise with intensity depending on biasing voltage. We have shown that Lorentzian noise originates from multilevel random telegraph noise of the small number of fluctuators which influence the tunneling process. Time-domain analysis of the current noise measured for temperatures between $4.2 \mathrm{~K}$ and $50 \mathrm{~K}$ enabled to determine the thermal activation energies of these fluctuators lying between 0.8 and $3 \mathrm{meV}$.
\end{abstract}

PACS: 73.50.Td, 73.40.Gk

\section{Introduction}

Low-frequency noise measurement in semiconductor heterostructures is a good alternative tool to investigate the kinetics of charge carriers being captured by localized states and/or released to delocalized band states. The generation-recombination noise arises when current flows through the sample and electron traps (defects/impurities), situated inside the heterostructure, can influence this process. Measuring the low-frequency spectral density of this noise and/or analysing time-series of 2or multilevel random telegraph noise (RTN) at different temperatures can give information about the energy of the barrier between the given two states of the fluctuator. It helps in identification of the defect/impurity in a heterostructure $[1,2]$. The present paper is a good example of the application of noise measurements for electrical characterization of heterostructure devices.

\section{Samples and measurement technique}

We investigated low-frequency tunnel current fluctuations in single-barrier GaAs/AlAs/GaAs heterostructures with $\mathrm{Si} \delta$-type doping in the AlAs barrier. The samples have been grown using MBE technique along the [100] crystallographic direction. The samples had a barrier width of $7 \mathrm{~nm}$ and an intentional planar concentration of Si impurity $1.5 \times 10^{9} \mathrm{~cm}^{-2}$. The impurities have been placed at a distance of $1 \mathrm{~nm}$ from the edge of the barrier - in one sample close to the substrate (sample $\# 1$ ), in another - close to the top contact (sample $\# 2$ ). The surface of mesas equaled $50 \times 50 \mu \mathrm{m}^{2}$ and $100 \times 100 \mu \mathrm{m}^{2}$, respectively.
The current noise has been measured by means of cross-correlation method with two independent transimpedance home-made amplifiers with gain $10^{7}$ and $10^{8} \mathrm{~V} / \mathrm{A}[3,4]$. Analysis of the current data has been performed in Matlab ${ }^{\circledR}$. All presented measurements have been made inside a liquid helium container and temperature was changed from $4.2 \mathrm{~K}$ to $50 \mathrm{~K}$ shifting the sample above the liquid helium level. We measured the current noise power spectral density for tunneling currents from $c a .10 \mathrm{pA}$ to $c a .1 \mu \mathrm{A}$. Applying a biasing voltage to the sample shifts the Fermi energy level in the emitter region close to the barrier relative to different states inside the barrier (also impurity and defect states). This results in the observation of different (localized) electron states contributing to tunnel current $[5,6]$.

\section{Results and discussion}

Usually the $I(V)$ characteristics (especially when differentiated) reported for similar single-barrier $\delta$-doped structures exhibit the features related to resonant tunneling [7]. However, despite the fact that the barrier is Si $\delta$-doped for both samples, for sample $\# 1$ we do not observe such features at all (Fig. 1). Moreover, in this sample the current density is much higher than in another one, doped close to the top contact (Fig. 1). This asymmetry is probably related to the interdiffusion of Si-donors during the growth process which smears the distribution of donors into the whole AlAs barrier in sample $\# 1$.

Figure 2 shows that not only the current but also the noise is much higher in sample \#1. This noise is not the 


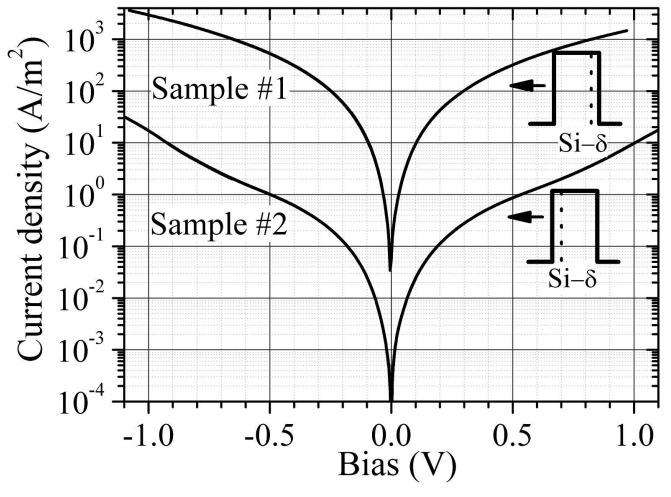

Fig. 1. The $I(V)$ characteristics of both measured samples. Arrows indicate the growth direction. Positive bias voltage means plus of voltage source applied to the top contact of the mesa.

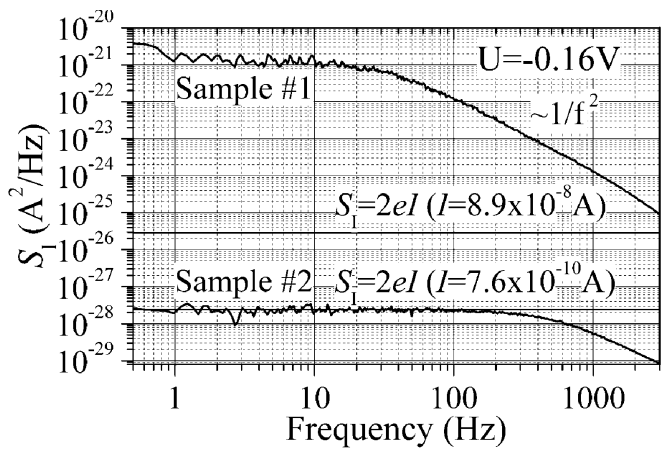

Fig. 2. Power spectral density of current fluctuations for two measured samples. Two horizontal lines indicate the full Schottky shot noise for currents flowing through the samples at a given bias $U=-0.16 \mathrm{~V}$. For sample \#2 the measured noise is equal to $2 e I$ - the full Schottky shot noise.

shot noise like in the case of sample \#2 but exceeds its value by more than four orders of magnitude. This noise spectrum has a Lorentzian character with $1 / f^{2}$ high-frequency roll-off (the observed decrease of noise spectral density above $200 \mathrm{~Hz}$ and $2 \mathrm{kHz}$ for sample \#2 and sample $\# 1$, respectively, is due to high-frequency cut-off of transimpedance amplifier for $10^{8}$ and $10^{7} \mathrm{~V} / \mathrm{A}$ gains). As it is clear from the current-signal in time-domain shown for sample $\# 1$ in Fig. 3 a, this Lorentzian originates from RTN. The analysis of the RTN presented in Fig. 3b enables to calculate average times spent by a fluctuator in upper and lower states $\left(\tau_{\text {up }}\right.$ and $\left.\tau_{\text {down }}\right)[8,9]$. Residual signal obtained after subtraction of traces (a) and (b) demonstrates that there are more than one fluctuator responsible for the observed signal (Fig. 3c).

Figure 4 presents the Arrhenius plot of average times spent by a fluctuator in upper and lower states $\left(\tau_{\text {up }}\right.$ and $\tau_{\text {down }}$ ) as determined from clean RTN signal from Fig. $3 \mathrm{~b}$. It enables to calculate the thermal activation energy of the fluctuator to be $E=2.3 \mathrm{meV}$. This energy is very

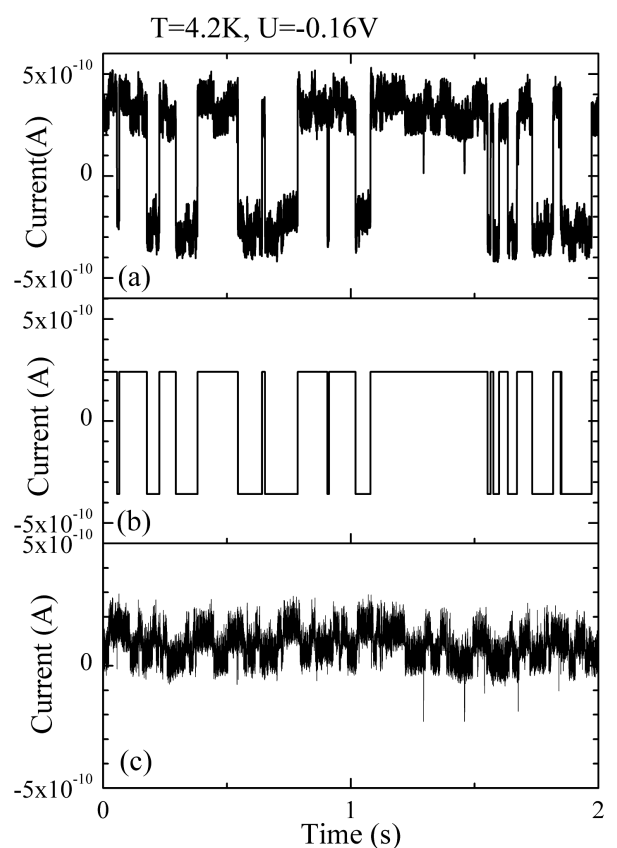

Fig. 3. Random telegraph noise for sample \#1 at bias voltage $U=-0.16 \mathrm{~V}$ : (a) as measured, (b) cleaned RTN signal after computer analysis $[8,9]$, (c) residual signal after subtraction (a)-(b).

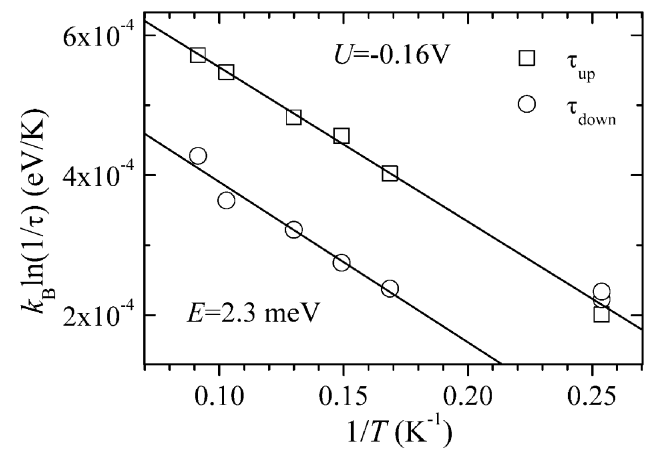

Fig. 4. Arrhenius plot of average times spent by a fluctuator in upper and lower states $\left(\tau_{\text {up }}\right.$ and $\left.\tau_{\text {down }}\right)$ as calculated from clean RTN signal in Fig. 3b.

small in comparison with other observations basing on noise measurements in similar materials [10-14]. It is too small to be just related to $\mathrm{Si}$ shallow donor states either in GaAs or in AlAs. However, the Coulomb potentials generated by remote ionized Si-donors placed in AlAs barrier create the local spatial potential fluctuations in GaAs emitter region which can probably temporarily localize electrons thus influencing the current flowing through the device. This could be possible mechanism of the observed RTN with so small activation energies. It should be stressed that random telegraph noise is clearly seen only for certain ranges of bias voltages and changing the bias causes the evolution of Lorentzian noise spectra and reveals different fluctuators which are 
observed as 2-, 3- or multilevel RTN and have thermal activation energes in the range $0.8-3 \mathrm{meV}$. It is in favor of above suggested interpretation since it could be related with the energetic structure of the potential fluctuations which trap the carriers.

\section{Conclusions}

Both investigated samples with $\mathrm{Si} \delta$-doping close to the edges of the barrier have similar shape of $I(V)$ characteristics. However, probably due to the diffusion of $\mathrm{Si}$ dopants during the growth process, in the case of effectively thinner barrier (sample \#1) we observed higher current densities and the RTN modulation noise related to trapping and detrapping of charges in local spatial potential fluctuations generated by remote impurities. For intentionally very similar structure (sample $\# 2$ ) with $\delta$ -doping on the other side of the barrier we obtained barrier with small transmission coefficient and white shot noise with Fano factor close to 1.

Our noise measurements enabled to have more insight into the electron transport mechanism than simple $I(V)$ characteristics and proved to be a powerfull tool for characterization of transport properties of nanostructures.

\section{Acknowledgments}

Work has been partially supported by the Polish Ministry of Science and Higher Education project No. N N202 192534 .

\section{References}

[1] M.J. Kirton, M.J. Uren, Adv. Phys. 38, 367 (1989).

[2] Sh. Kogan, Electronic Noise and Fluctuations in Solids, Cambridge University Press, Cambridge 1996.

[3] J. Przybytek, M. Baj, Acta Phys. Pol. A 112, 221 (2007).

[4] J. Przybytek, M. Baj, Am. Inst. Phys. Conf. Proc. 1129, 333 (2009).

[5] M. Gryglas, M. Baj, B. Chenaud, B. Jouault, A. Cavanna, G. Faini, Phys. Rev. B 69, 165302 (2004).

[6] B. Jouault, M. Gryglas, G. Faini, U. Gennser, A. Cavanna, M. Baj, D.K. Maude, Phys. Rev. B 73, 155415 (2006).

[7] S. Lewińska, M. Gryglas-Borysiewicz, J. Przybytek, M. Baj, U. Gennser, A. Ouerghi, Acta Phys. Pol. A 119, 606 (2011).

[8] Y. Yuzhelevski, M. Yuzhelevski, G. Jung, Rev. Sci. Instrum. 71, 1681 (2000).

[9] G. Giusi, F. Crupi, C. Pace, Rev. Sci. Instrum. 79, 024701 (2008).

[10] T. Judd, N.R. Couch, P.H. Beton, M.J. Kelly, T.M. Kerr, M. Pepper, Appl. Phys. Lett. 49, 1652 (1986).

[11] M.H. Weichold, S.S. Villareal, R.A. Lux, Appl. Phys. Lett. 55, 657 (1989).

[12] Sze-Him Ng, Ch. Surya, E.R. Brown, P.A. Maki, Appl. Phys. Lett. 62, 2264 (1993).

[13] S.Y. Tzeng, M.J. Cich, R. Zhao, H. Feick, E.R. Weber, Appl. Phys. Lett. 82, 1063 (2003).

[14] R. Khlil, A. El Hdiy, Y. Jin, J. Appl. Phys. 98, 093709 (2005). 